\title{
A Call for Appropriate Evidence and Outcomes-Based Use and Measurement of Anticoagulation for Atrial Fibrillation: Moving the Population Towards Improved Health Via Multiple Stakeholders
}

\author{
David S. Kountz, MD, MBA; Fadia T. Shaya, PhD, MPH; Alan H. Gradman, MD; Gary A. Puckrein, PhD; \\ Michael H. Kim, MD; Jennifer Wilbanks, PharmD; James G. Stevenson, PharmD, FASHP; \\ David L. Larsen, RN, MHA; Michael Wysong, BS; Viktor Chirikov, PhD, MS; \\ Wayne T. Pan, MD; and Liou Xu, PhD
}

\begin{abstract}
SUMMARY
A multidimensional approach involving consideration of available resources, individual patient characteristics, patient preferences, and cost of treatment is often required to optimize clinical decision making in the management of atrial fibrillation (AF). In order to bring together varying perspectives on effective tactics and to formulate innovative strategies to improve the management of AF, a think tank consortium of advisors was assembled from across the spectrum of health care stakeholders. Focus groups were conducted and facilitated by a moderator and a notetaker. Participants were asked to comment on preliminary data for the increased prevalence of $\mathrm{AF}$, patterns of treatment, impact of adherence with anticoagulants on clinical and economic outcomes, and opportunities for optimizing treatment.

Several recommendations to reach short- and long-term goals in improving AF management emerged from the focus group discussions. These recommendations specifically targeted 3 stakeholder groupspatients/caregivers, physicians, and payers-and addressed the need for better understanding of determinants of undertreatment and nonadherence for those on anticoagulation therapy. Recommendations included the use of real-world data studies to understand regional and demographic patterns of treatment and outcomes, the development of an enhanced national quality standard for anticoagulation, and engaging patients in shared decision making to optimize satisfaction with treatment. Actionable strategies were presented to address gaps related to anticoagulation management. Balancing new anticoagulants' higher prescription costs and safety concerns with their superior effectiveness and convenience of administration for at-risk individuals would require a concerted effort involving patients and their caregivers, physicians, and payers.
\end{abstract}

J Manag Care Spec Pharm. 2015;21(11):1034-38

Copyright $\odot 2015$, Academy of Managed Care Pharmacy. All rights reserved.

A trial fibrillation (AF) is a common underdiagnosed and undertreated cardiac arrhythmia characterized by uncoordinated atrial activation, deterioration of atrial mechanical function, and an irregular pulse. Its presence reduces exercise tolerance, promotes the development of heart failure, and is associated with a 2- to 7 -fold increase in stroke risk and a near doubling of all-cause mortality., ${ }^{1,2}$ As the most frequently encountered cardiac arrhythmia in clinical practice affecting almost 3 million Americans, AF needs to be treated to reduce mortality- and morbidity-associated costs. ${ }^{3,4}$ The risk of $\mathrm{AF}$ increases with age, and its prevalence is rising throughout the industrialized world. Census-based projections estimate that by the year 2050, the number of individuals in the United States with this disorder will increase to between 12 and 15 million individuals. ${ }^{3,5}$ These facts and the rising cost burden associated with preventive therapy and, more importantly, the care of AF complications highlight the need for more clinical and cost-effective treatments of this common condition.

The dramatic increase in stroke, which occurs in patients with $\mathrm{AF}$, is related to thrombus formation usually in the left atrial appendage. In patients with AF, uncoordinated left atrial contraction results in sluggish blood flow (stasis), which predisposes to thrombosis. The embolization of these thrombi and the subsequent obstruction of blood vessels in the brain account for approximately $15 \%$ of all strokes that occur in the United States. ${ }^{5}$ Clinical trials have established that anticoagulation with warfarin reduces the risk of stroke by more than $60 \%$ in patients with nonvalvular AF. ${ }^{6}$ Although highly effective, warfarin is difficult to use for many patients. In addition, large segments of the AF population do not receive any treatment at all.

Recently, a number of advances have occurred in the preventive care of patients with AF. Innovative techniques are being designed to maintain warfarin anticoagulation levels consistently within the therapeutic range. In addition, a new class of nonvitamin $\mathrm{K}$ antagonist oral anticoagulants (NOACs) has been introduced. These agents offer certain safety advantages over warfarin and do not require continuous monitoring of the level of anticoagulation - a factor that may be beneficial in promoting adherence to therapy. However, these agents are significantly more expensive than warfarin, and their anticoagulation effect may not be readily reversible in certain circumstances. Furthermore, the management of AF still presents treatment gaps with regards to certain subpopulations and regions. ${ }^{8}$ Selection of the most appropriate therapy in individual patients is often challenging. A multidimensional approach involving consideration of available resources, individual patient characteristics, patient preferences, and cost of treatment is often required to optimize clinical decision making.

\section{Description of Focus Groups}

In order to bring together varying perspectives on effective tactics to improve the management of AF patients, the Atrial 


\section{A Call for Appropriate Evidence and Outcomes-Based Use and Measurement of Anticoagulation for Atrial Fibrillation: Moving the Population Towards Improved Health Via Multiple Stakeholders}

Fibrillation Think Tank Consortium was organized and met in Houston, Texas, on October 17, 2014. The assembled 25 advisors included leaders from across the spectrum of managed care and included academicians and physicians and directors, chief officers, and executives of managed care organizations, accountable care organizations, integrated delivery networks, integrated hospital systems, hospitals, pharmacies, and medical societies. Structured discourse and focus groups were held with a goal of formulating innovative strategies to better manage anticoagulation in AF populations served by managed care organizations. What follows is a summary of these discussions.

The conduct of the focus groups was facilitated by a moderator and a notetaker. The discussions were centered on an analysis done by the National Minority Quality Forum, a nonpartisan research and educational organization dedicated to ensuring that high-risk and ethnic populations and communities receive optimal health care. ${ }^{9}$ This unpublished analysis, which used Medicare administrative claims data, was characterized by several major findings: (a) an increased prevalence of AF among Medicare enrollees from 2000 to 2010, attributed to improved survival; (b) crisis consumers (4\%) and heavy consumers (27\%) of health services covered disproportionately $27 \%$ and $51 \%$ of overall AF costs; (c) treated patients had lower average annual cost per patient as well as lower annual all-cause hospitalization and morality rates; (d) only $24 \%$ of patients were compliant on anticoagulation therapy (lower numbers were applicable to warfarin and NOACs); and (e) AF prevalence and nonadherence to anticoagulation therapies, analyzed cumulatively, exhibited geographical cluster patterns with more than three quarters of AF cases occurring in less than $25 \%$ of U.S. ZIP codes, with New England states having the lowest rates of compliance to warfarin.

Participants were asked to comment on this preliminary data on the increased prevalence of $\mathrm{AF}$, patterns of treatment, impact of adherence with anticoagulants on clinical and economic outcomes, and opportunities for optimizing treatment. Given the controversy regarding how to balance new anticoagulants' higher prescription costs and safety concerns with their superior effectiveness and convenience of administration for at-risk individuals, the focus of discussions was on identifying gaps relevant to anticoagulation management and suggesting sound strategies to address those gaps.

\section{Summary of Discussions}

Several themes emerged from the focus group discussions. Overall, participants saw an opportunity to understand and disseminate information regarding the primary and secondary prevention effects of anticoagulation therapy, in terms of preventing acute events and of more general health outcomes.

\section{Undertreatment and Nonadherence}

An interesting discussion point was that, possibly unknown to decision makers, a large number of AF patients may not be on treatment or may have low levels of adherence with anticoagulation therapy. Focus group participants partly attributed undertreatment of AF to the lack of awareness among physicians of the high costs associated with not managing AF. While discussants perceived that any level of adherence to AF therapy seemed beneficial, they interpreted presented study results to highlight more improved benefits at higher levels of adherence. Participants agreed that there was a lack of clarity on the determinants of nonadherence for those on therapy and who the untreated patients are and why they are not treated. There was a strong perceived need for well-conducted real-world studies to understand AF treatment patterns and outcomes, differences between various treatment options, and related regional or other demographic variations.

\section{Geo-Coded Information}

Much of the focus group discussions centered on creating interactive maps to elucidate factors of underlying patient behavior and cues to action in high-risk AF patient subpopulations. Using ZIP code-level retrospective data to create geographic maps showing trends for AF prevalence, costs, and adherence in high-risk African-American and rural subpopulations, for example, could help identify untreated and noncompliant patients and develop patient profiles for different communities. Such visualized geo-coded information could also be useful in exploring geographic clusters with higher than average numbers of crisis consumers and in partnering with payers in those areas to devise and deliver targeted educational messages and management strategies.

\section{Quality Measures}

There was much discussion regarding the need for a better, well-accepted quality measure for anticoagulation management and therapy at the population level. In particular, discussions were driven by a sense of urgency and support for the development of a national AF quality standard specific to anticoagulation management in AF patients. While a list of anticoagulation quality of care metrics put forth by the Agency for Healthcare Research and Quality, the Joint Commission, and the National Quality Forum do exist, ${ }^{10}$ participants deemed that existing quality metrics did not accurately represent the nature of AF management and associated health outcomes. For example, the National Quality Forum metric, NQF 1525, is submitted by physician practices to report anticoagulation therapy prescription rates among high-risk adults diagnosed with AF or atrial flutter, but the metric does not require that prescription rates be linked to achieving certain health outcomes or clinical thresholds. Focus group participants suggested that features of the new quality measure should include, at a minimum, the definition of acceptable rates of AF patients on anticoagulation therapy; the proportion of patients achieving some minimal 
level of adherence to anticoagulation therapy; and the rates of AF patients who have been assigned $\mathrm{CHA}_{2} \mathrm{DS}_{2}$-VASc scores, an AF severity index for stroke risk. Discussants viewed several organizations as instrumental partners to engage in discussions on the development of such quality metrics. These organizations included the Medicare Star Ratings system, the National Committee for Quality Assurance, Pharmacy Quality Alliance, National Quality Forum, and Choosing Wisely Campaign from the ABIM Foundation.

\section{Cost}

Increased cost to patients was also seen by focus group participants as an important factor in perceived reluctance among physicians to prescribe NOACs, even as discussants acknowledged NOACs to have a better safety profile for patients. To address cost barriers, it was suggested that innovative coverage and reimbursement models were needed. Those items would capitalize on pharmacoeconomic data specific to payers' populations and could be tied to a new well-established anticoagulation therapy quality measure. In particular, advisors perceived payers as reluctant to expand coverage and reimbursement for NOACs unless the use of those drugs was covered under risk-based contracting strategies incorporating enhanced quality measures related to readmissions or other Medicare Star metrics.

\section{Shared Decision Making}

Finally, a major part of the focus group discussions revolved around the need to address a potential discrepancy between patient preferences and physician choice of prescribed anticoagulant. For example, while NOACs may present an improved safety and effectiveness profile for generally sicker or nonadherent patients, about two thirds of focus group participants viewed the current rates of NOAC prescribing and use to be suboptimal. However, participants unanimously agreed that patient engagement should be a major focus. With the availability of multiple options of anticoagulation therapy, a shared decision-making process, embraced by physicians and patients, would optimize satisfaction with treatment and treatment adherence rates.

\section{Recommendations}

Overall, several recommendations to reach short- and longterm goals for improving AF management emerged from the focus group discussions. These recommendations specifically targeted 3 stakeholder groups: patients and caregivers, physicians, and payers.

\section{Patients and Caregivers}

In the short term, based on the premise that greater adherence with anticoagulant therapy is beneficial, discussants thought that copay strategies might improve patient access to treatment. Furthermore, providing easy-to-understand and culturally appro- priate AF educational material to those undiagnosed or untreated patients living in geographic clusters with elevated rates of AF prevalence could increase the awareness of the importance of treatment and adherence in improving AF outcomes.

In the long term, it was deemed that future initiatives should concentrate on refining educational support tools for disease awareness and shared decision making, especially for patients and physicians residing in high-risk geographic areas, and ensuring that any educational messages delivered be succinct and synchronized across the dissemination channels of pharmaceutical manufacturers, providers, payers, and retailers. Likewise, future development of quality indicators to measure success in the management of AF should be subject to easy interpretation by patients and should be tied to new patient access and health plan structures that incentivize patients to stay compliant with therapy. As such, innovative value-based benefit designs and patient access programs are needed.

\section{Physicians}

In the short term, focus group findings suggest increasing awareness by physicians of the high prevalence of untreated AF patients via educational sessions and promoting better screening practices to primary care physicians and the wider spectrum of AF providers. This was thought to be particularly critical in geographic areas with high numbers of AF patients who are not treated. Additionally, it was suggested that education sessions should be centered on the theme of shared decision making with the patient and should include topics on eliciting patient preferences in order to increase adherence, how to effectively communicate clinical information on NOACs effectiveness and safety to the patient, and how to use a patient lifestyle selector tool. One means of delivering the proposed education program to providers could include developing innovative continuing education courses led by credible unbiased thought leaders in the field. Furthermore, dissemination of the latest clinical guidelines and interactive discussion of patient cases among physicians may be achieved via social networking platforms.

In the long term, besides further developing and refining educational activities to providers, physicians were seen by advisors as a central stakeholder group engaged in the development of a quality measure on AF anticoagulation therapy and management, providing input on how such a measure can adequately respond to the reality of strained resources in the health system. Furthermore, enlisting physician support to transform anticoagulation clinics into full-service anticoagulation clinics that would include NOAC-treated patients as well as warfarintreated patients was deemed an effective means to decrease physician concern for prescribing NOACs in that the clinics would provide an additional point of service and management of patients with $\mathrm{AF}$. 


\section{A Call for Appropriate Evidence and Outcomes-Based Use and Measurement of Anticoagulation for Atrial Fibrillation: Moving the Population Towards Improved Health Via Multiple Stakeholders}

\section{Payers}

In the short term, a natural conclusion derived from the focus group discussions was that payers should be made aware of the existence of geographic clusters of untreated patients and the importance of maintaining adherence to anticoagulation medication to prevent high-cost use, such as preventable hospitalizations. As previously mentioned, developing contracting strategies that leverage geographic information was seen by discussants as instrumental in increasing patient access to medications, while minimizing the risk of disproportionally enrolling high-risk users. Further consideration of risk-based market access programs was also deemed important.

The reliance of pharmacy benefit managers (PBM) exclusively on data specific to the population covered by the health plan, however, was raised as a potential limitation to using regional and national geographic data to influence a PBM formulary decision-making process. Nevertheless, priority actionable areas for PBMs, such as using well-established real-world evidence on improved adherence with NOACs and meeting a nationally recognized quality measure standard for anticoagulation therapy and management, were considered important steps to alleviate barriers and advance the formulary decisionmaking process.

Interestingly, while payers have traditionally not been involved in the shared decision-making process between patient and physician, the consensus among focus group participants was that this important health care stakeholder group could play an integral role in delivering information on patientcentered AF management to patients and physicians. Providing education on the importance of shared decision making could favorably affect the process of matching the wide availability of anticoagulation options to a patient's individual profile and preferences. It was deemed that the alignment of a patient's convenience and lifestyle factors with treatment characteristics may significantly increase compliance and reduce the need for ordering laboratory tests and recalibrating dosing schedules.

In the long term, it was suggested that medication therapy management (MTM) programs, which are increasingly showing favorable impact on adherence and health outcomes, should be more regularly integrated into the services offered by health plans. Further discussions focused on leveraging the pharmacist workforce for MTM to manage anticoagulation therapy in transitions between care settings.

\section{Conclusions}

The analysis of focus group discussions pointed to a potentially existing awareness gap among payers regarding the favorable cost impact of anticoagulation therapies. With the focus on big data and availability of well-linked and rich patient-level datasets, pharmacoeconomic studies are becoming more relevant, detailed, and able to evaluate the impact of various treatment patterns and regimens on specific subpopulations of interest to payers. For example, educating payers at managed care conferences on the use of well-conducted pharmacoeconomic analyses was seen as 1 practical venue in which to encourage pharmacy managers to invest resources to acquire new analytic tools or pursue innovative medication access partnerships.

\section{Authors}

DAVID S. KOUNTZ, MD, MBA, is Vice President, Academic Affairs, Jersey Shore University Medical Center, Neptune, New Jersey. FADIA T. SHAYA, PhD, MPH, is Professor and Vice Chair for Academic Affairs, and VIKTOR CHIRIKOV, PhD, MS, is Associate Scientist, Pharmaceutical Health Services Research, University of Maryland School of Pharmacy, Baltimore. ALAN H. GRADMAN, MD, is Professor of Medicine, Temple University School of Medicine (Clinical Campus), Pittsburgh, Pennsylvania; MICHAEL H. KIM, MD, is Professor of Medicine, Warren Alpert Medical School of Brown University, Providence, Rhode Island; JENNIFER WILBANKS, PharmD, is Adjunct Assistant Professor, Ferris State University, Novi, Michigan; JAMES G. STEVENSON, PharmD, FASHP, is Professor, College of Pharmacy, University of Michigan, Ann Arbor; DAVID L. LARSEN, RN, MHA, is Director, Quality Improvement, Select Health, Murry, Utah; MICHAEL WYSONG, $B S$, is Chief Executive Officer, CARE Pharmacies Cooperative, Linthicum, Maryland; and WAYNE T. PAN, MD, is Chief Medical Officer, Applied Research Works, Palo Alto, California. GARY A. PUCKREIN, PhD, is President and Chief Executive Officer, and LIOU XU, PhD, is Biostatistician, National Minority Quality Forum, Washington, $D C$.

AUTHOR CORRESPONDENCE: Fadia T. Shaya, PhD, MPH, Professor and Vice Chair for Academic Affairs, University of Maryland School of Pharmacy, 220 Arch St., 12th Fl., Rm. 01-204, Baltimore, MD 21201. Tel.: 410.706.5392; E-mail: fshaya@rx.umaryland.edu.

\section{DISCLOSURES}

This study was supported by Daiichi Sankyo. Stevenson and Wysong received consulting fees from Inside Edge Consulting. Kim received consulting fees from Novosys Health and has received consulting fees from Boehringer Ingelheim. Kim has also served as advisory board member for DaiichiSankyo. Pan is a consultant for Daiichi Sankyo and Inside Edge Consulting. Wilbanks, Puckrein, and Xu received funding from Daiichi-Sankyo.

All authors contributed equally to the concept and design of this commentary, as well as to the gathering of data and the writing and revision of the manuscript.

\section{ACKNOWLEDGMENTS}

Inside Edge Consulting Group provided editorial assistance for the development of the manuscript. 


\section{A Call for Appropriate Evidence and Outcomes-Based Use and Measurement of Anticoagulation for Atrial Fibrillation: Moving the Population Towards Improved Health Via Multiple Stakeholders}

\section{REFERENCES}

1. Dulli DA, Stanko H, Levine RL. Atrial fibrillation is associated with severe acute ischemic stroke. Neuroepidemiology. 2003;22(2):118-23.

2. Benjamin EJ, Wolf PA, D’Agostino RB, Silbershatz H, Kannel WB, Levy D. Impact of atrial fibrillation on the risk of death: the Framingham Heart Study. Circulation. 1998;98(10):946-52.

3. Go AS, Hylek EM, Phillips KA, et al. Prevalence of diagnosed atrial fibrillation in adults: national implications for rhythm management and stroke prevention: the AnTicoagulation and Risk Factors in Atrial Fibrillation (ATRIA) Study. JAMA. 2001;285(18):2370-75.

4. Kim MH, Johnston SS, Chu BC, Dalal MR, Schulman KL. Estimation of total incremental health care costs in patients with atrial fibrillation in the United States. Circ Cardiovasc Qual Outcomes. 2011;4(3):313-20.

5. Go AS, Mozaffarian D, Roger VL, et al. Heart disease and stroke statistics-2013 update: a report from the American Heart Association. Circulation. 2013;127(1):e6-e245.
6. Hart RG, Pearce LA, Aguilar MI. Meta-analysis: antithrombotic therapy to prevent stroke in patients who have nonvalvular atrial fibrillation. Ann Intern Med. 2007;146(12):857-67.

7. Kakkar AK, Mueller I, Bassand JP, et al. Risk profiles and antithrombotic treatment of patients newly diagnosed with atrial fibrillation at risk of stroke: perspectives from the international, observational, prospective GARFIELD registry. PLoS One. 2013;8(5):e63479.

8. Yancy CW, Wang TY, Ventura HO, et al. The coalition to reduce racial and ethnic disparities in cardiovascular disease outcomes (credo): why credo matters to cardiologists. J Am Coll Cardiol. 2011;57(3):245-52.

9. National Minority Quality Forum. Website. Available at: http://www. nmqf.org/. Accessed September 23, 2015.

10. Eymin G, Jaffer AK. Evidence behind quality of care measures for venous thromboembolism and atrial fibrillation. J Thromb Thrombolysis. 2014:37(2):87-96 